\title{
Imaging Biomarkers in Ischemic Stroke Clinical Trials: An Opportunity for Rigor
}

T he effective treatment of major strokes due to major cerebral artery occlusion is a great opportunity for diagnostic and interventional neuroradiology. These represent approximately onethird of all strokes and result in the most deaths and poor outcomes. The relevant physiology (occlusion site, infarct core, and penumbra) is revealed by neuroimaging, and many patients, perhaps most, are effectively and safely treatable by neurointerventional methods. After an early success, there were many clinical trial failures. However, there are indications of a major breakthrough with the positive results reported by the completed A Randomized Trial of Intraarterial Treatment for Acute Ischemic Stroke $^{1}$ and in early interim analyses of other trials. While highly encouraging, the good outcome rate of $<33 \%$ suggests that there are opportunities for improvement. The role of imaging biomarkers in enhancing this opportunity looms large. However, optimal patient outcomes will only be achieved if the neuroradiology community uses rigor in the application of stroke imaging biomarkers.

There are several possible reasons for prior clinical trial failures. One factor was poorly effective devices for vessel recanalization. Recently introduced devices appear to have largely resolved this problem. The remaining major obstacle is patient selection. In a review published in this issue of the American Journal of Neuroradiology, Harston et $\mathrm{al}^{2}$ provide a timely and valuable service by documenting the problem of heterogeneity in the types of imaging biomarkers heretofore used in stroke trials. They suggested that this is an important reason for the failure of many of these trials. They further suggest that more homogeneity in the use of imaging biomarkers would be better. While the article covers much important ground, it does fully explore why such heterogeneity has resulted in repeated, though not uniform, failures in clinical trials.

There are at least 3 reasons why imaging biomarkers fail:

1) The wrong measurement has been chosen

2) The measurement criterion is mistaken

3) There are significant errors in making the measurement.

To illuminate these 3 factors, one should consider what the authors identify as the most common biomarker used in stroke trials: the measurement of the core infarct volume to exclude pa- tients who are unlikely to benefit from a treatment or intervention. There is strong evidence that this is an appropriate measure. If a large infarct is already present, arterial recanalization may be futile and possibly harmful by risking hemorrhage, reperfusion injury, or malignant edema. Nevertheless, many trials have failed despite the use of this measure. This brings up reason 2: Was the wrong infarct size selected? To help explore this possibility, one should consider an absurd example- exclusion of only those patients who have an identifiable infarct that is the size of the entire MCA territory or larger. Clearly, it would be difficult to show that a treatment is beneficial because so many patients would be admitted into the trial who would have no possibility of benefiting.

What is the best measurement criterion for infarct size to use as a threshold? As noted in the article, a core infarct involving one-third of the MCA territory $(\sim 100 \mathrm{~mL}$ ) has been the most commonly used threshold, but there is now substantial evidence that this volume threshold is probably too high. The multicenter Diffusion and Perfusion Imaging Evaluation for Understanding Stroke Evolution 2 trial $^{3}$ demonstrated that patients presenting with DWI lesion volumes of $<70 \mathrm{~mL}$ had better outcomes when undergoing endovascular treatment. This lower volume threshold was also found to lead to better outcomes in 3 single-center studies. ${ }^{4-6}$ Evidence that one-third of the MCA territory is too large also comes from the failed Mechanical Retrieval and Recanalization of Stroke Clots Using Embolectomy trial, ${ }^{7}$ which used a threshold of $90 \mathrm{~mL}$. Thus, perhaps an infarct involving "one-third of the MCA territory" is wrong.

The final possible reason for poor patient selection is that the imaging method produces errors that are too large to be adequate for the task. Again consider core infarct size. Does the chosen imaging biomarker measure the core infarct with sufficient precision to be useful? There is general agreement with much evidence that unenhanced CT is inadequate for this purpose. There is also general agreement with strong animal and human evidence that DWI does, in fact, reliably measure the core infarct. On the other hand, no animal studies have shown that a single hemodynamic measurement corresponds to infarcted tissue. Moreover, CTP-derived images have inherently poor signal-to-noise ratios, which result in large measurement errors. ${ }^{8}$ Perhaps it would be wise to hold off on the use of perfusion CT (PCT) in stroke trials 
until it has been rigorously validated in animal stroke models and measurement errors are reduced.

Against this logic is the clear preference for the use of PCT over DWI as is evident in the review by Harston et al. ${ }^{2}$ The principal justification is that PCT is widely available and readily accessible to patients with stroke, while MR imaging is much less so. Thus, it appears that this decision is based on "practicality" rather than scientific evidence. This possibility raises important ethical questions. Should we use a less effective tool because it is practical, or should we make the necessary effort to make available to our patients with stroke the tool that clearly benefits them?

Rigorous standards for validation of imaging biomarkers are missing, and they are unlikely to be forthcoming without a demand for them by the stroke clinical research community. Such rigor would likely result in the abandonment of imaging biomarkers that perform poorly. However, the other result would be progress toward more homogeneity in the use of imaging biomarkers, and ultimately better outcomes in patients with acute stroke whom we treat.

\section{REFERENCES}

1. Berkhemer OA, Fransen PS, Beumer D, et al. A randomized trial of intraarterial treatment for acute ischemic stroke. N Engl J Med 2015; 372:11-20
2. Harston GW, Rane, N, Shaya S, et al. Imaging biomarkers in acute ischemic stroke trials: a systematic review. AJNR Am J Neuroradiol 2015;36:839-43

3. Lansberg MG, Straka M, Kemp S, et al. MRI profile and response to endovascular reperfusion after stroke (DEFUSE 2): a prospective cohort study. Lancet Neurol 2012;11:860-67

4. Simonsen CZ, Sorensen LH, Karabegovic S, et al. MRI before intraarterial therapy in ischemic stroke: feasibility, impact, and safety. J Cereb Blood Flow Metab 2014;34:1076-81

5. Wisco D, Uchino K, Saqqur M, et al. Addition of hyperacute MRI AIDS in patient selection, decreasing the use of endovascular stroke therapy. Stroke 2014;45:467-72

6. Yoo AJ, Verduzco LA, Schaefer PW, et al. MRI-based selection for intraarterial stroke therapy: value of pretreatment diffusion-weighted imaging lesion volume in selecting patients with acute stroke who will benefit from early recanalization. Stroke 2009;40:2046-54

7. Kidwell CS, Jahan R, Gornbein J, et al. A trial of imaging selection and endovascular treatment for ischemic stroke. $N$ Engl $\mathrm{J} \mathrm{Med}$ 2013;368:914-23

8. Schaefer PW, Souza L, Kamalian S, et al. Limited reliability of computed tomographic perfusion acute infarct volume measurements compared with diffusion-weighted imaging in anterior circulation stroke. Stroke 2015;46:419-24

R.G. González Massachusetts General Hospital and Harvard Medical School Boston, Massachusetts 